\title{
Towards a Dialogue System that Supports Rich Visualizations of Data
}

\author{
Abhinav Kumar and Jillian Aurisano and Barbara Di Eugenio and Andrew Johnson \\ University of Illinois at Chicago \\ Chicago, IL USA \\ \{akumar34,jauris2,bdieugen,ajohnson\}@uic.edu
}

\author{
Alberto Gonzalez and Jason Leigh \\ University of Hawai'i at Manoa \\ Honolulu, HI USA \\ \{agon,leighj\}@ hawaii.edu
}

\begin{abstract}
The goal of our research is to support fullfledged dialogue between a user and a system that transforms the user queries into visualizations. So far, we have collected a corpus where users explore data via visualizations; we have annotated the corpus for user intentions; and we have developed the core NL-to-visualization pipeline.
\end{abstract}

\section{Introduction}

Visualization, even in its simplest forms, remains a highly effective means for converting large volumes of raw data into insight. Still, even with the aid of robust visualization software, e.g. Tableau ${ }^{1}$ and ManyEyes (Viegas et al., 2007), especially novices face challenges when attempting to translate their questions into appropriate visual encodings (Heer et al., 2008; Grammel et al., 2010). Ideally, users would like to tell the computer what they want to see, and have the system intelligently create the visualization. However, existing systems (Cox et al., 2001; Sun et al., 2013; Gao et al., 2015) do not offer two-way communication, or only support limited types of queries, or are not grounded in how users explore data.

Our goal is to develop Articulate 2, a fullfledged conversational interface that will automatically generate visualizations. The contributions of our work so far are: a new corpus unique in its genre ${ }^{2}$ and a prototype system, which is able to process a sequence of requests, create the corresponding visualizations, position them on the screen, and manage them.

\footnotetext{
${ }^{1}$ http://www.tableau.com/

${ }^{2}$ The corpus will be released at the end of the project.
}

\section{Related Work}

Much work has focused on the automatic generation of visual representations, but not via NL (Feiner, 1985; Roth et al., 1994; Mackinlay et al., 2007). Likewise, much work is devoted to multimodal interaction with visual representations (e.g. (Walker et al., 2004; Meena et al., 2014)), but not to automatically generating those visual representations. Systems like AutoBrief (Green et al., 2004) focus on producing graphics accompanied by text; or on finding the appropriate graphics to accompany existing text (Li et al., 2013).

(Cox et al., 2001; Reithinger et al., 2005) were among the first to integrate a dialogue interface into an existing information visualization system, but they support only a small range of questions. Our own Articulate (Sun et al., 2013) maps NL queries to statistical visualizations by using very simple NLP methods. When DataTone (Gao et al., 2015), the closest to our work, cannot resolve an ambiguity in an NL query, it presents the user with selection widgets to solve it. However, only one visualization is presented to the user at a given time, and previous context is lost. (Gao et al., 2015) compares DataTone to IBM Watson Analytics, ${ }^{3}$ that allows users to interact with data via structured language queries, but does not support dialogic interaction either.

\section{A new corpus}

15 subjects, 8 male and 7 female, interacted with a remote Data Analysis Expert (DAE) who assists the subject in an exploratory data analysis task: analyze crime data from 2010-2014 to provide suggestions as to how to deploy police officers in four neighborhoods in Chicago. Each session consisted of multiple cycles of visualization construc-

\footnotetext{
${ }^{3}$ http://www.ibm.com/analytics/watson-analytics/
} 


\begin{tabular}{lc}
\hline & DAE Communication Types \\
\hline 1. & Greeting \\
\hline 2. & Clarification \\
\hline 3. & Correction \\
\hline 4. & Specified data not found \\
\hline 5. & Can do that \\
\hline 6. & Cannot do that \\
\hline 7. & Done \\
\hline
\end{tabular}

Table 1: DAE Communication Types

tion, interaction and interpretation, and lasted between 45 and 90 minutes.

Subjects were instructed to ask spoken questions directly to the DAE (they knew the DAE was human, but couldn't make direct contact ${ }^{4}$ ). Users viewed visualizations and limited communications from the DAE on a large, tiled-display wall. This environment allowed analysis across many different types of visualizations (heat maps, charts, line graphs) at once (see Figure 1).

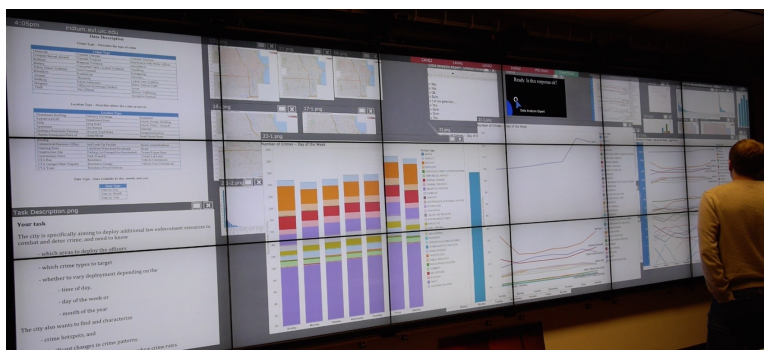

Figure 1: A subject examining crime data.

The DAE viewed the subject through two highresolution, direct video feeds, and also had a mirrored copy of the tiled-display wall on two $4 \mathrm{~K}$ displays. The DAE generated responses to questions using Tableau, and used SAGE2 (Marrinan et al., 2014), a collaborative large-display middlewear, to drive the display wall. The DAE could also communicate via a chat window, but confined herself to messages of the types specified in Table 1. Apart from greetings, and status messages (sorry, it's taking long) the DAE would occasionally ask for clarifications, e.g. Did you ask for thefts or batteries. Namely, the DAE never responded with a message, if the query could be directly visualized; neither did the DAE engage in multi-turn elicitations of the user requirements. Basically, the DAE tried to behave like a system with limited dialogue capabilities would.

Table 2 shows summary statistics for our data, that was transcribed in its entirety. So far, we

\footnotetext{
${ }^{4}$ In a strict Wizard-of-Oz experiment, the subjects would not have been aware that the DAE is human.
}

\begin{tabular}{ccc}
\hline Words & Utterances & Directly Actionable Utts. \\
\hline 38,105 & 3,179 & 490 \\
\hline
\end{tabular}

Table 2: Corpus size

have focused on the type of requests subjects pose. Since no appropriate coding scheme exists, we developed our own. Three coders identified the directly actionable utterances, namely, those utterances ${ }^{5}$ which directly affect what the DAE is doing. This was achieved by leaving an utterance unlabelled or labeling it with one of 10 codes ( $\kappa=0.84$ (Cohen, 1960) on labeling an utterance or leaving it unlabeled; $\kappa=0.74$ on the 10 codes). The ten codes derive from six different types of actionable utterances, which are further differentiated depending on the type of their argument. The six high-level labels are: requests to create new visualizations (8\%, e.g. Can I see number of crimes by day of the week?), modifications to existing visualizations $(45 \%, U m m$, yeah, I want to take a look closer to the metro right here, umm, a little bit eastward of Greektown); window management instructions (12.5\%, If you want you can close these graphs as I won't be needing it anymore); factbased questions, whose answer doesn't necessarily require a visualization (7\%, During what time is the crime rate maximum, during the day or the night?); requests for clarification (20.5\%, Okay, so is this statistics from all 5 years? Or is this for a particular year?); expressing preferences (7\%, The first graph is a better way to visualize rather than these four separately).

Three main themes have emerged from the analysis of the data. 1) Directly actionable requests cover only about $15 \%$ of what the subject is saying; the remaining $85 \%$ provides context that informs the requests (see Section 6). 2) Even the directly actionable $15 \%$ cannot be directly mapped to visualization specifications, but intermediate representations are needed. 3) An orthogonal dimension is to manage the visualizations that are generated and positioned on the screen.

So far, we have made progress on issues 2) and 3). The NL-to-visualization pipeline we describe next integrates state-of-the-art components to build a novel conversational interface. At the moment, the dialogue initiative is squarely with the user, since the system only executes the requests. However, strong foundations are in place

\footnotetext{
${ }^{5}$ What counts as an utterance was defined at transcription.
} 
for it to become a full conversational system.

\section{The NL-to-visualization pipeline}

The pipeline in Figure 2 illustrates how Articulate 2 processes a spoken utterance, first by translating it into a logical form and then into a visualization specification to be processed by the Visualization Executor (VE). For create/modify visualization requests, an intermediate SQL query is also generated.

Before providing more details on the pipeline, Figure 3 presents one example comprising a sequence of four requests, which results in three visualizations. The user speaks the utterances to the system by using the Google Speech API. The first utterance asks for a heatmap of the "River North" and "Loop" neighborhoods (two downtown areas in Chicago). The system generates the visualization in the upper-left corner of the figure. In response to utterance $b$, Articulate 2 generates a new visualization, which is added to the first visualization (see bottom of screen in the middle); it is a line graph because the utterance requests the aggregate temporal attribute "year", as we discuss below. The third request is absent of aggregate temporal attributes, and hence the system produces a bar chart also added to the display. Finally, for the final request $d$ ), the system closes the most recently generated visualization, i.e. the bar chart (this is not shown in Figure 3).

\subsection{Parsing}

We begin by parsing the utterance we obtain from the Google Speech API into three NLP structures. ClearNLP (Choi, 2014) is used to obtain PropBank (Palmer et al., 2005) semantic role labels (SRLs), which are then mapped to Verbnet (Kipper et al., 2008) and Wordnet using SemLink (Palmer, 2009). The Stanford Parser is used to obtain the remaining two structures, i.e. the syntactic parse tree and dependency tree. The final formulation is the conjunction $C_{\text {predicate }} \cap C_{\text {agent }} \cap$ $C_{\text {patient }} \cap C_{\text {det }} \cap C_{\text {mod }} \cap C_{\text {action. }}$. The first three clauses are extracted from the SRL. The NPs from the syntactic parse tree contain the determiners for $C_{d e t}$, adjectives for $C_{m o d}$, and nouns as arguments for $C_{\text {action }}$.

\subsection{Request Type Classification}

A request is classified into the six actionable types mentioned earlier, for which we developed a mul-

\begin{tabular}{cc}
\hline Feature Type & Total Terms \\
\hline Trigrams & 3,203 \\
\hline Bigrams & 2,311 \\
\hline Tagged Unigrams & 784 \\
\hline Unigrams & 584 \\
\hline Head word & 314 \\
\hline Part-of-Speech & 33 \\
\hline Chunks & 15
\end{tabular}

Table 3: Feature Types

ticlass classifier. We applied popular question classification features from (Loni et al., 2011) due to the general question-based construct of the requests. Apache OpenNLP (Apache Software Foundation, 2011) was used to generate unigrams, bigrams, trigrams, chunking, and tagged unigrams, while Stanford Parser's implemented Collins rules (Collins, 2003) were used to obtain the headword. The feature vector is comprised of 7,244 total features, see Table 3. We used Weka (Hall et al., 2009) to experiment with several classifiers. We will discuss their performance in Sec. 5; currently, we use the SVM model, which performs the best.

\subsection{Window Management Requests}

If the classifier assigns to an utterance the window management type, a logical form along the lines described above will be generated, but no SQL query will be produced. At the moment, keyword extraction is used to determine whether the window management instruction relates to closing, opening, or repositioning; the system only supports closing the most recently created new visualization.

\subsection{Create/Modify Visualization Requests}

If the utterance is classified as a request to create or modify visualizations, the logical form is used to produce an SQL query. ${ }^{6}$ SQL was partly chosen because the crime data we obtained from the City of Chicago is stored in a relational database.

Most often, in their requests users include constraints that can be conceptualized as standard filter and aggregate visualization operators. In utterance $c$ in Figure 3, assaults can be considered as a filter, and location as an aggregator (location is meant as office, restaurant, etc.). We distinguish between filter and aggregate based on types stored in the $\mathrm{KO}$, a small domain-dependent

\footnotetext{
${ }^{6}$ Since our system does not resolve referring expressions yet, currently all visualization requests result in a new visualization.
} 


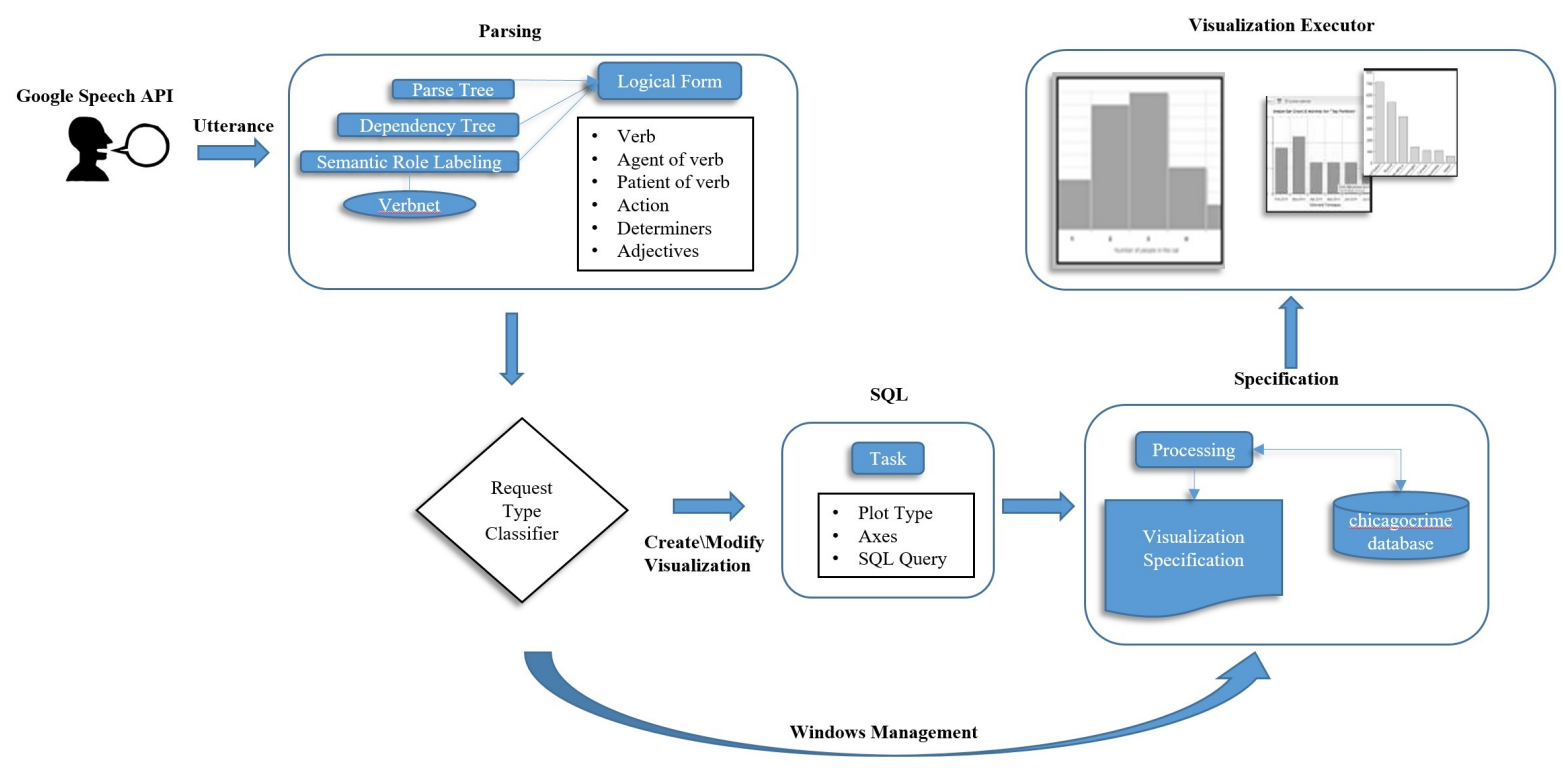

Figure 2: NL-to-Visualization Pipeline

knowledge ontology. ${ }^{7}$ The KO contains relations, attributes, and attribute values. Filters such as "assault" are defined as attribute values in the KO, whereas aggregate objects such as "location" are attribute names. A synonym lexicon contains synonyms corresponding to each entry in the KO. SQL naturally supports these operators, since the data can be filtered using the "WHERE" clause and aggregated with the "GROUP BY" clause.

\subsection{Vizualization Specification}

The final transformation is from SQL to visualization specification. Overall, the specification for creating a new visualization includes the $\mathrm{x}$-axis, $y$-axis, and plot type. Finally, the VE uses Vega (Trifacta, 2014) to plot a graphical representation of the visualization specification on SAGE2. We currently support 2-D bar charts, line graphs, and heat maps. The different representations for sentence $c$ ) from Figure 3 are shown here:

Logical Form: see.01(a) $\cap$ Action(a, Loop, assault, location) $\cap \operatorname{Det}(\mathrm{a}$, the $)$

SQL: SELECT count(*) as TOTAL_CRIME, location FROM chicagocrime WHERE (neighborhood = loop) AND (crimetype $=$ assault) GROUP BY location

Visualization Specification: \{"horizontalAxis": "NON_UNIT", "horizontalGroupAxis": "location", "verticalAxis": "TOTAL_CRIME", "plotType": "BAR"\}

\footnotetext{
${ }^{7}$ The system is re-configurable for different domains by updating the $\mathrm{KO}$.
}

\section{Evaluation}

Since the work is in progress, a controlled user study cannot be carried out until all the components of the system are in place. We have conducted piecemeal smaller and/or informal evaluations of its components. For example, we have manually inspected the results of the pipeline on the 38 requests that concern creating new visualizations. The pipeline produces the correct SQL expression (that is, the actual SQL that a human would produce for a given request) for 31 (81.6\%) of them (spoken features such as filled pauses and restarts were removed, but the requests are otherwise processed unaltered). The seven unsuccessful requests fail for various reasons, including: two are fact-based that cannot be answered yet; two require mathematical operations on the data which are not currently supported; one does not have a main verb, one does not name any attributes or values (can I see the map - in the future, our conversational interface will ask for clarification). For the last one, the SQL query is generated, but it is very complex and the system times out.

As concerns classifying the request type, Table 4 reports the results of the classifiers trained on the features discussed in Section 4.2. The SVM results are statistically significantly different from the Naive Bayes results (paired $t$-test), but indistinguishable from Random Forest or Multinomial Naive Bayes.

As concerns the whole pipeline, our prelimi- 


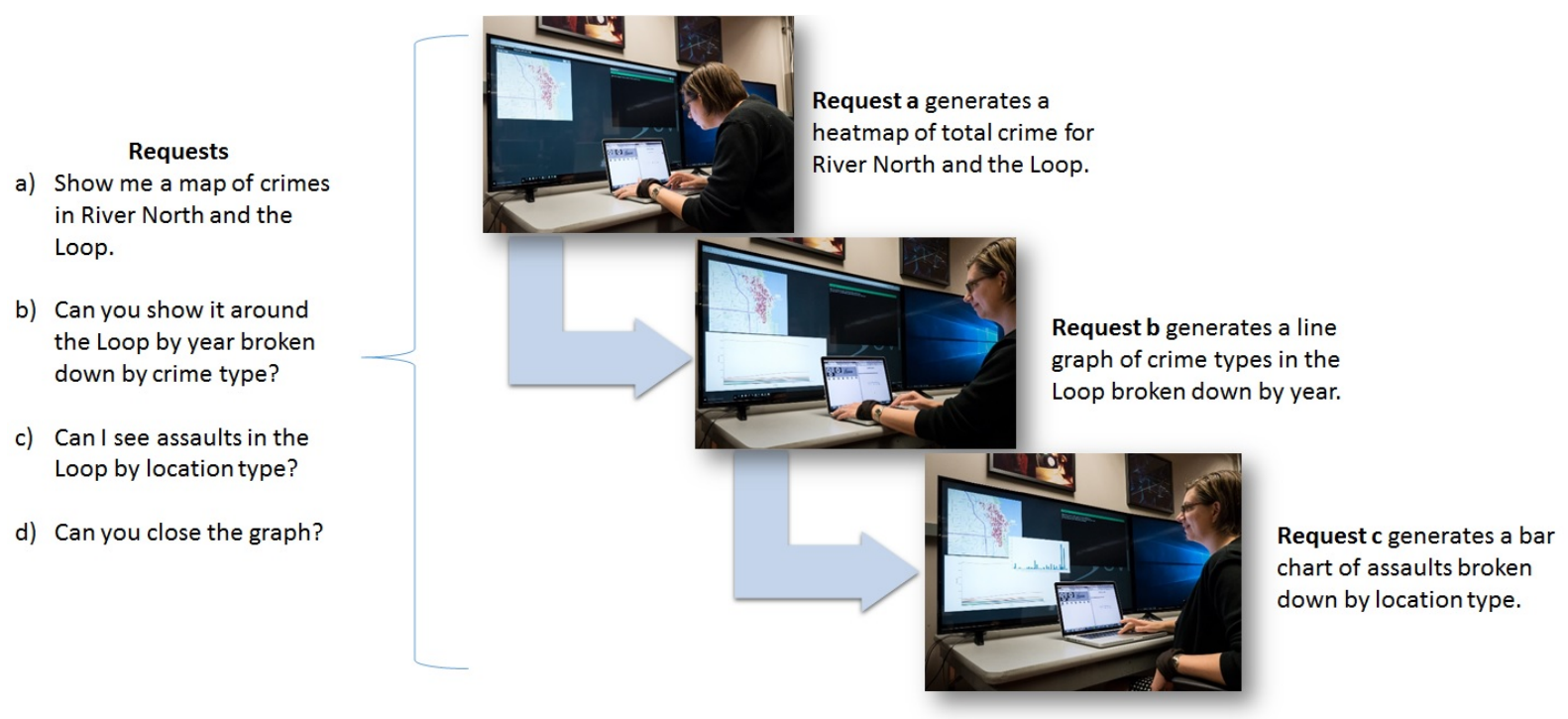

Figure 3: Incremental generation of visualizations

\begin{tabular}{lc}
\hline Classifier & Accuracy \\
\hline Support Vector Machines & $87.65 \%$ \\
\hline Random Forest & $85.60 \%$ \\
\hline Multinomial Naive Bayes & $85.60 \%$ \\
\hline Naive Bayes & $74.28 \%$ \\
\hline
\end{tabular}

Table 4: Request Type Classification Accuracy

nary, informal observation is that the generated visualization specifications result in accurate and appropriate visualizations. However, we have not dealt with constraints across visualizations: e.g., consistent application of colors by attribute (theft is always blue), would help users integrate information across visualizations.

\section{Current Work}

Annotation. We are focusing on referring expressions (see below), and on the taxonomy of abstract visualization tasks from (Brehmer and Munzner, 2013). This taxonomy, which includes why a task is performed, will help us analyze that $85 \%$ of the users utterances that are not directly actionable. In fact, many of those indicate the why, i.e. the user's goal (e.g., "I want to identify the places with violent crimes.").

Dialogue Manager / Referring Expressions. We are developing a Dialogue Manager (DM) and a Visualization Planner (VP) that will be in a continuous feedback loop. If the DM judges the query to be unambiguous, it will pass it to the VP. If not, the DM will generate a clarification request for the user. We will focus on referring expression resolution, necessary when the user asks for a modification to a previous visualization or wants to manipulate a particular visualization. In this domain, referring expressions can refer to graphical elements or to what those graphical elements represent (color the short bar red vs. color the theft bar red), which creates an additional dimension of coding, and an additional layer of ambiguity.

The Visualization Planner. The VP both needs to create more complex visualizations, and to manage the screen real estate when several visualizations are generated (which is the norm in our data collection, see Figure 1, and reflected in the system's output in Figure 3). The VP will determine the relationships between the visualizations on screen and make decisions about how to position them effectively. For instance, if a set of visualizations are part of a time series, they might be more effective if ordered on the display.

\section{References}

Apache Software Foundation. 2011. Apache OpenNLP. http://opennlp.apache.org.

Matthew Brehmer and Tamara Munzner. 2013. A multi-level typology of abstract visualization tasks. IEEE Transactions on Visualization and Computer Graphics, 19(12):2376-2385.

Jinho D. Choi. 2014. Optimization of Natural Language Processing components for Robustness and Scalability. Ph.D. thesis, University of Colorado Boulder.

Jacob Cohen. 1960. A coefficient of agreement for nominal scales. Educational and Psychological Measurement, 20:37-46. 
Michael Collins. 2003. Head-driven statistical models for natural language parsing. Computational Linguistics, 29(4):589-637.

Kenneth Cox, Rebecca E Grinter, Stacie L Hibino, Lalita Jategaonkar Jagadeesan, and David Mantilla. 2001. A multi-modal natural language interface to an information visualization environment. International Journal of Speech Technology, 4(3-4):297314.

Steven Feiner. 1985. APEX: an experiment in the automated creation of pictorial explanations. IEEE Computer Graphics and Applications, 5(11):29-37.

Tong Gao, Mira Dontcheva, Eytan Adar, Zhicheng Liu, and Karrie G. Karahalios. 2015. DataTone: Managing Ambiguity in Natural Language Interfaces for Data Visualization. In Proceedings of the 28th Annual ACM Symposium on User Interface Software \& Technology, pages 489-500. ACM.

Lars Grammel, Melanie Tory, and Margaret Anne Storey. 2010. How information visualization novices construct visualizations. IEEE Transactions on Visualization and Computer Graphics, 16(6):943-952.

Nancy L. Green, Giuseppe Carenini, Stephan Kerpedjiev, Joe Mattis, Johanna D. Moore, and Steven F. Roth. 2004. Autobrief: an experimental system for the automatic generation of briefings in integrated text and information graphics. Int. J. Hum.-Comput. Stud., 61(1):32-70.

Mark Hall, Eibe Frank, Geoffrey Holmes, Bernhard Pfahringer, Peter Reutemann, and Ian H. Witten. 2009. The WEKA data mining software: an update. ACM SIGKDD Explorations Newsletter, 11(1):10 18.

Jeffrey Heer, Frank Van Ham, Sheelagh Carpendale, Chris Weaver, and Petra Isenberg. 2008. Creation and collaboration: Engaging new audiences for information visualization. In Information Visualization, pages 92-133. Springer.

Karin Kipper, Anna Korhonen, Neville Ryant, and Martha Palmer. 2008. A large-scale classification of English verbs. Language Resources and Evaluation, 42(1):21-40.

Zhuo Li, Matthew Stagitis, Kathleen F. McCoy, and Sandra Carberry. 2013. Towards Finding Relevant Information Graphics: Identifying the Independent and Dependent Axis from User-Written Queries. In Proceedings of the Twenty-Sixth International Florida Artificial Intelligence Research Society Conference (FLAIRS 13), pages 226-231.

Babak Loni, Gijs Van Tulder, Pascal Wiggers, David MJ Tax, and Marco Loog. 2011. Question classification by weighted combination of lexical, syntactic and semantic features. In Text, Speech and Dialogue, pages 243-250. Springer.
Jock D. Mackinlay, Pat Hanrahan, and Chris Stolte. 2007. Show me: Automatic presentation for visual analysis. IEEE Transactions on Visualization and Computer Graphics, 13(6):1137-1144.

Thomas Marrinan, Jillian Aurisano, Arthur Nishimoto, Krishna Bharadwaj, Victor Mateevitsi, Luc Renambot, Lance Long, Andrew Johnson, and Jason Leigh. 2014. Sage2: A new approach for data intensive collaboration using scalable resolution shared displays. In Collaborative Computing: Networking, Applications and Worksharing (CollaborateCom), pages 177-186. IEEE.

Raveesh Meena, Gabriel Skantze, and Joakim Gustafson. 2014. Data-driven models for timing feedback responses in a Map Task dialogue system. Computer Speech \& Language, 28(4):903-922.

Martha Palmer, Daniel Gildea, and Paul Kingsbury. 2005. The Proposition Bank: An Annotated Corpus of Semantic Roles. Computational Linguistics, 31(1):71-105, March.

Martha Palmer. 2009. Semlink: Linking PropBank, Verbnet and FrameNet. In Proceedings of the Generative Lexicon Conference, pages 9-15.

Norbert Reithinger, Dirk Fedeler, Ashwani Kumar, Christoph Lauer, Elsa Pecourt, and Laurent Romary. 2005. MIAMM - A Multimodal Dialogue System Using Haptics. In J. van Kuppevelt, L. Dybkjaer, and N. O. Bernsen, editors, Advances in Natural Multimodal Dialogue Systems, pages 307-332. Springer.

Steven F Roth, John Kolojejchick, Joe Mattis, and Jade Goldstein. 1994. Interactive graphic design using automatic presentation knowledge. In Proceedings of the SIGCHI Conference on Human Factors in Computing Systems, pages 112-117. ACM.

Yiwen Sun, Jason Leigh, Andrew Johnson, and Barbara Di Eugenio. 2013. Articulate: Creating Meaningful Visualizations from Natural Language. In Weidong Huang and Mao Lin Huang, editors, Innovative Approaches of Data Visualization and Visual Analytics, pages 218-235. IGI Global.

Trifacta. 2014. Vega: A Visualization Grammar. https://vega.github.io/vega/.

Fernanda B. Viegas, Martin Wattenberg, Frank Van Ham, Jesse Kriss, and Matt McKeon. 2007. ManyEyes: a Site for Visualization at Internet Scale. IEEE Transactions on Visualization and Computer Graphics, 13(6):1121-1128.

M.A. Walker, S.J. Whittaker, A. Stent, P. Maloor, J. Moore, M. Johnston, and G. Vasireddy. 2004. Generation and evaluation of user tailored responses in multimodal dialogue. Cognitive Science, 28(5):811-840. 